\title{
Diseño inmaterial - Hacia la desmaterialización y digitalización de productos y servicios como herramienta de sostenibilidad
}

\author{
Rivera-Pedroza, Julio César ${ }^{a}$ y Hernandis-Ortuño, Bernabéb \\ ${ }^{a} \mathrm{PhD}$ candidate at Universitat Politècnica de València, Spain. - Investigador y diseñador industrial de la Universidad \\ Nacional de Colombia. juriped@doctor.upv.es; juceriv@hotmail.com. \\ ${ }^{\mathrm{b}} \mathrm{PhD}$. Full Professor. Universitat Politècnica de València, Spain. bhernand@.upv.es
}

\begin{abstract}
Resumen
La crisis ambiental es también un problema de comportamiento, y no está limitado sólamente a tecnología, producción y volumen. Por lo tanto, con la evolución y los avances en la tecnología, los procesos y métodos de producción para desarrar nuevos productos y servicios, es necesario analizar el nuevo papel del diseño en la sociedad actual. El objetivo propuesto de este estudio es el de describir la relación entre dinámicas de desmaterialización y digitalización (o informacionalización) de productos y servicios y la sostenibilidad.
\end{abstract}

La investigación se lleva a cabo a partir de un estudio de caso con un enfoque cualitativo y un énfasis analítico-descriptivo, sobre la manera en que factores tales como los avances tecnológicos, junto con los comportamientos y las emociones de los usuarios influyen en la configuración de productos y servicios y su relación con la sostenibilidad. Es importante resaltar hasta qué punto se puede sentir amenazado el "know how" del diseñador con la aparición, cada vez más frecuente, de productos y servicios desmaterializados y/o digitalizados. En este sentido, se plantea que, más que una amenaza, puede ser una oportunidad para evolucionar, considerando enfoques sistémicos desde una perspectiva multi-objetivo, multidimensional y multidisciplinaria. El presente análisis podría proporcionar pistas en el campo del diseño, teniendo en cuenta el contexto inmaterial para desarrollar productos y servicios desmaterializados y digitalizados, comprometidos con una sociedad más sostenible.

Palabras clave: Diseño, inmaterial, desmaterialización, digitalización, sostenibilidad.

\footnotetext{
Abstract

The environmental crisis is also a behavioral issue, and not one simply of technology, production, and volume. Thus, with the evolution and advances in technology, processes and production methods for development of new products, it is necessary to analyze the new role of design in today's society. The proposed aim of this paper is to describe the relationship between dematerialization and digitalization (or informationalization) of products and services into sustainability.
} 
Diseño inmaterial. Hacia la desmaterialización y digitalización de productos y servicios como herramienta de sostenibilidad.

The research is carried out from a case study with a qualitative approach and an analyticaldescriptive emphasis, how factors such as technological advances along with behavior and emotions of users influence the configuration of products and services and its relationship to sustainability. It is important to stress to what extent the "know how" from the Designer can feel threatened with the appearance of increasingly frequent dematerialized and/or digitalized products and services. And in this sense, it might be stated that rather than a threat, it could be an opportunity to evolve, considering systemic approaches from a multi-objective, multidimensional and multidisciplinary perspective. This analysis could provide clues to field of knowledge taking into account the immaterial context, to develop dematerialized and/or digitalized products and services committed with a more sustainable society.

Keywords: Design, immaterial, dematerialization, digitalization, sustainability.

\section{Introducción}

La actividad del Diseño tiene una gran responsabilidad del actual estado del medio ambiente, además de un papel fundamental en la búsqueda de la sostenibilidad. En el contexto actual, enfocado hacia una sociedad sostenible, es conveniente reflexionar sobre la influencia que tiene el diseño sobre productos y servicios, a modo de lograr un planteamiento, desarrollo y puesta en funcionamiento -o uso- de los mismos de manera sostenible. Del mismo modo, es necesario reflexionar sobre la forma de abordar los problemas de la sostenibilidad, pues se considera que aunque ya se han logrado algunos avances, es un campo aún por explorar. De hecho, hasta hace poco, las metodologías de diseño sostenible raramente estaban comprometidas con las cuestiones más fundamentales como el sentido y el lugar de los productos y servicios en nuestras vidas, y la contribución de los bienes materiales a lo que podría ser definido ampliamente como el esfuerzo humano (Chapman, 2009). Aunque las cuatro décadas y media de actividades del diseño sostenible, según y cómo lo afirma Chapman, "han hecho este desperdicio e ineficiencia ligeramente menos derrochadora e ineficiente" (2009, pág. 30), es una perspectiva de sostenibilidad limitada e insuficiente desde un punto de vista evolutivo y de proyección en el tiempo, por lo que se hace indispensable un abordaje desde otras perspectivas, en términos de proponer alternativas y medios que permitan alcanzar una sociedad sostenible en todos los niveles.

La sostenibilidad está evolucionando, actualmente su naturaleza va más allá de los tres pilares básicos con que era concebida (ecológico, económico y social), por lo que es conveniente analizar si hay una nueva visión de la sostenibilidad, en la cual a través de una perspectiva holística y sistémica de las alteraciones ambientales, sea posible encontrar soluciones que incluyan elementos materiales e inmateriales relacionados con el comportamiento humano y las dimensiones culturales. Planteamientos propuestos por autores como Walker (2006), Wahl \& Baxter (2008), González (2013) o Wigum (2004), destacan la importancia de las motivaciones esenciales de los individuos, como una la fuerza dinámica que permita un cambio en productos y servicios, a través de las demandas y aspiraciones reales, que no pueden llevarse a cabo y definirse sólo a través de hechos físicos.

Autores como Mugge, Schoormans, \& Schifferstein (2007) se cuestionan sobre el porqué las personas desarrollan relaciones sólidas hacia determinados productos y cómo los diseñadores pueden influir en el grado de apego a través del diseño de productos. Aunque lo anterior se refiere a productos tangibles, se 
plantea que también se puede presentar en productos y servicios intangibles. Lo cual es una gran oportunidad para que, diseñadores y desarrolladores de productos y servicios, lo enfoquen hacia su área de estudio, y en éste caso en especial hacia "la sostenibilidad".

Por otro lado, también es importante tener en cuenta al usuario o consumidor y su percepción sobre la sostenibilidad, a modo de identificar cuales son los rasgos, aspectos o atributos que deben poseer productos y servicios sostenibles, según sus criterios. Esto puede servir para identificar, cómo se le puede dar un nuevo enfoque a la sostenibilidad -en caso de ser necesario- o comprobar si el modelo actual es el adecuado; con el fin de ratificar o replantear el concepto que la sociedad tiene de la sostenibilidad. Para ello se debe ir más allá de la caracterización del fenómeno. Es decir, saber si el fenómeno de la sostenibilidad hoy en día se caracteriza por reparación, reuso, uso secundario, mínimo consumo de recursos, recuperación, reciclaje, compostaje, etc., o si se está caracterizando por otros aspectos basados en actualización, cambio de formato (producto a servicio), desmaterialización, sustitución, virtualización, multifuncionalidad, optimización de la vida útil, uso compartido, creación de experiencias, vinculo emocional y otros elementos que podrían hacer parte de ese contexto inmaterial, relacionado con las emociones y los valores, y que posiblemente no sean considerados en la actualidad como impulsores de la sostenibilidad.

El propósito del actual estudio es, desde una perspectiva sistémica, reflexionar sobre unas dimensiones material (contexto material) e inmaterial (contexto inmaterial), que se creen presentes en el planteamiento, desarrollo y puesta en funcionamiento -o uso- de productos y servicios, a modo de identificar un diseño inmaterial que relacione las actuales dinámicas de desmaterialización y digitalización -o informacionalización- de productos y servicios con la sostenibilidad. Con base en lo mencionado, se plantea el análisis de un estudio de caso en el que se consideren enfoques y estudios basados en las necesidades de los seres humanos, así como las emociones y sistemas de valores, que permitan reconocer puntos clave pertenecientes a esa dimensión inmaterial, los cuales a menudo se pasan por alto en la configuración de un producto o servicio y que se cree son relevantes al momento de generar una solución de diseño sostenible.

\section{Marco conceptual}

\subsection{El modelo de diseño concurrente}

El presente estudio se basa en el Modelo de Diseño Concurrente (MDC) de Hernandis, B. (2003). El modelo consta principalmente de un sistema exterior y de un sistema de referencia o sistema en estudio. En el sistema exterior se consideran tanto los aspectos relacionados con la dimensión más tangible del diseño del producto/servicio (materias primas, procesos, tecnologías, funcionalidad, distribución, proveedores, infraestructuras, entre otros), así como los aspectos más próximos a una dimensión intangible (cultura, sociedad, emociones y valores de los usuarios, percepción y motivaciones de las personas, entre otros) y demás aspectos que aportan consideraciones y restricciones que influyen sobre el problema de diseño. 
Diseño inmaterial. Hacia la desmaterialización y digitalización de productos y servicios como herramienta de sostenibilidad.

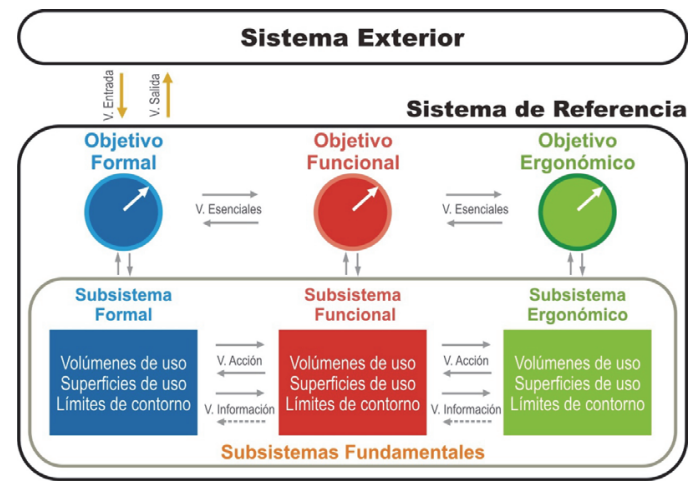

Fig. 1 Modelado teórico. Fuente: adaptado de Hernandis (2003)

Se parte de la idea de que en el sistema exterior se encuentran los suprasistemas (o subsistemas del sistema exterior), que abarcan la realidad que nos rodea y pueden definir las variables que permiten la configuración de un producto, sistema o proceso; y es en ésta fase del diseño conceptual en la que se deben aplicar los criterios para generar una respuesta sostenible a un problema planteado. Desde esta perspectiva, Vezzoli \& Manzini (2008, pág. 238) afirman que, "mejorar el impacto de productos es más probable durante las primeras fases de desarrollo, cuando la innovación tiene una mayor magnitud".

\subsection{Derivación del sistema exterior}

Cualquier problema de diseño, abordado desde la sistémica, se debe asumir como un sistema que se compone de subsistemas en donde la respuesta, acertada o no, depende de las interacciones y relaciones de estos subsistemas o componentes, en donde, según Wahl \& Baxter (2008), es pertinente abarcar otra dimensión, además de la física, para obtener respuestas acertadas. En este sentido, existen dos extremos específicos: el primero, a partir de artefactos culturales, instituciones, patrones de producción y consumo, que expresan la intencionalidad material; y el segundo, en la dimensión inmaterial, el "metadiseño" de nuestro conocimiento consciente, sistemas de valores, cosmovisiones y aspiraciones que definen la intencionalidad detrás del diseño materializado.

Se propone una perspectiva de sostenibilidad apoyada en una vision holistica y sistémica, que abarque varias disciplinas, panoramas y enfoques, que permitan y faciliten una acertada toma de decisiones. Para ello Rivera et al (2013), plantean dos contextos como componentes del sistema exterior, el contexto material y el contexto inmaterial, a manera de reconocer criterios que validen los supuestos o conocimientos sobre los conceptos identificados de la realidad percibida.

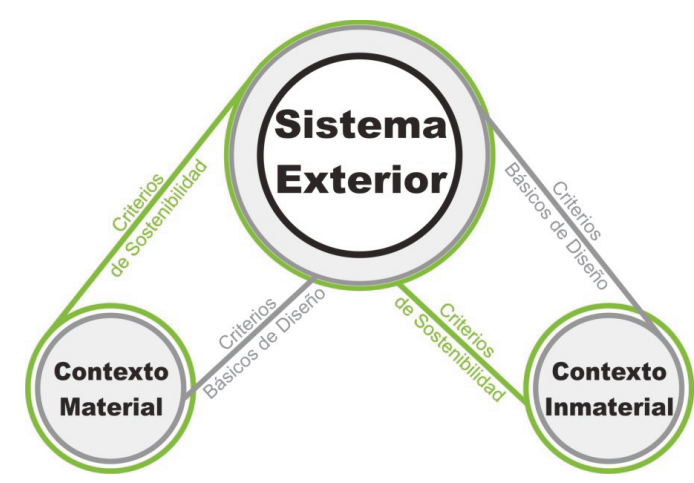

Fig. 2 Esquema de derivación del Sistema Exterior. Fuente: adaptado de Rivera et al (2013) 
Resultado de estos análisis se ha identificado, que además de las variables de entrada, relacionadas con el contexto material de un problema de diseño, hay otras asociadas con un contexto inmaterial en el que se consideran aspectos emocionales y valores, como factores psicológicos y psicosociales, que satisfacen necesidades no materiales de los usuarios/consumidores.

\subsubsection{Contexto material}

En el contexto material, se suponen aspectos ligados a los conceptos físicos de productos y servicios, en donde son analizadas las características, materiales, producción, energía, etc., además de las relaciones e interacciones de elementos ya desarrollados y el medio en que se utilizan. A este respecto, Wahl \& Baxter (2008), indican que la intencionalidad que hay materialmente detrás del diseño, "se expresa a través de las interacciones y relaciones formadas por productos de consumo, sistemas de transporte, economías, sistemas de gobierno, patrones de asentamiento, y los recursos y la energía utilizados, con la complejidad de los procesos sociales y ecológicos (pág. 74). Bajo esta perspectiva, se propone que durante el planteamiento y desarrollo de una solución a un problema de diseño se debe realizar un "análisis físico" en el que se consideren aspectos relacionados con el componente tangible o contexto material del proyecto.

\subsubsection{Contexto inmaterial}

En este contexto, se formulan analisis relacionados con conceptos psicológicos y sociológicos que estén ligados a las diversas cosmovisiones, ideas, sistemas de valores y aspiraciones de la sociedad. Wahl \& Baxter (2008), señalan que, inmaterialmente nuestras ideas de organización, cosmovisiones y sistemas de valores, expresan cómo damos sentido a nuestra experiencia de la realidad a través del metadiseño (pág. 74). Aquí esta formación del sentido por medio del metadiseño, va más allá de los aspectos tangibles del contexto material, para lograr una relación con conceptos y supuestos psicológicos y sociológicos. En esa dirección, Stegall (2006) afirma, que el nuevo objetivo es diseñar productos que sean más que simplemente no tóxicos o reciclables, en realidad sirvan como herramientas para formar personas, vidas y valores, para lo cual es necesario examinar los rasgos, valores y comportamientos que las personas deben tener en una sociedad sostenible (pág. 58). Para ello, es necesario un enfoque holístico en el que se incluyan diversas disciplinas académicas y profesionales, visiones y enfoques diferentes.

Lo que se pretende con la derivación del sistema exterior, es observar en el contexto inmaterial, si algunos de sus componentes -necesidades, emociones y valores-, que a menudo son ignorados en la configuración de un producto o servicio, pueden ser relevantes al generar soluciones de diseño sostenible. A partir de este planteamiento, se analizan los anteriores contextos, para identificar elementos que estén en línea con la sostenibilidad, considerando teorías relacionadas con las necesidades humanas, las emociones y los valores, desde lo que se ha denominado diseño inmaterial, mediante dinámicas sostenibles emergentes de desmaterialización y digitalización de productos y servicios.

\subsection{Dinámicas sostenibles emergentes}

El diseño sostenible está madurando, se cree que hay un cambio hacia una nueva dimensión, en donde una serie de motivaciones (necesidades, emociones y valores) traen consigo una nueva visión de la sostenibilidad que pasa por la desmaterialización y digitalización de productos y servicios. En el Atlas del Diseñador de Sostenibilidad, Thorpe (2010) se refiere a esta mayoría de edad como la segunda etapa en un debate en el cual el rol del diseño en aspectos económicos y sociales de la sostenibilidad está más plenamente explorado, además de la atención ya establecidos en materia de energía y materiales (Thorpe, 2010, pág. 5). Para Chapman (2009), la crisis de la sostenibilidad es un problema de conducta, y no simplemente de tecnología, producción y volumen. Las condiciones de comportamiento que ambos 
Diseño inmaterial. Hacia la desmaterialización y digitalización de productos y servicios como herramienta de sostenibilidad.

manejan y los patrones de la influencia del consumo de materiales son complejos, pero fundamentales para un compromiso efectivo con una agenda contemporánea de diseño sostenible.

Afirmaciones como las anteriores hacen que emerjan interrogantes sobre las perspectivas de la sostenibilidad y los roles de cada uno de los actores involucrados en alcanzarla, así como del surgimiento de dinámicas sostenibles emergentes como la desmaterialización y la digitalización de productos y servicios que consoliden una nueva dimensión de sostenibilidad, en donde, y siguiendo a Robèrt et al (2002), se genere una transformación cultural que cambie el foco en los productos y servicios, a fin de encontrar completamente nuevas formas de satisfacer las mismas necesidades en los usuarios/consumidores, sean estas necesidades básicas o de autorealización.

\subsubsection{Desmaterialización}

En primer lugar, se harán algunas aclaraciones sobre el concepto de “desmaterialización” utilizado en el presente estudio, debido a que este término tiene varias interpretaciones. En este caso en particular, el termino desmaterialización es tomado como una estratégia que apoya a la sostenibilidad, con antecedentes asociados desde la Rueda de LiDs (Lifecycle Design Strategies) de Brezet \& van Hemel (1997) hasta enfoques más conteporaneos que podrían estar asociados al bienestar humano; tal como sugieren Beuren, Ferreira, \& Miguel (2013), quienes citando a Baines et al., (2007), indican que la desmaterialización de productos, además de haber sido discutida en la literatura por autores como Mont (2001), Ehrenfeld (2001), Manzini \& Vezzoli (2003), Wong (2004) y Tomiyama (2001), también ha sido utilizada como un objetivo para los sistemas producto-servicio (PSS en inglés product service-systems).

Li, Zhang, Li, \& Tong (2010), afirman que la desmaterialización se ha convertido en un concepto importante en la ecología industrial, el cual ha penetrado en todas las fases del ciclo de vida del producto. A lo que Beuren et al (2013) interpretan, que consecuencia de ello, un producto puede ser desmaterializado mediante la inclusión de servicios que reducen la cantidad de materiales consumidos en el ciclo de vida de un producto, no sólo en su creación sino también en su uso, reutilización y reciclaje; en ese sentido Kestemont \& Kerkhove (2010) aseveran, que la idea es tender hacia un desarrollo más sostenible y eficiente para "producir más bienestar humano utilizando menos recursos naturales", es decir, desvinculando el crecimiento económico del uso de material, mediante la utilización de menos "cosas", o en su defecto de productos y servicios más eficientes, proyectados y desarrollados desde la desmaterialización. En este caso, no se trataría solamente de la desmaterialización a través de la cantidad de material consumido, sino y como afirma Cleary (2010), con posibles escenarios de gestión de residuos, incluyendo la prevención de residuos, mediante la ampliación de los límites del sistema. Lo cual podría ser la prevención de residuos a razón de la desmaterialización, en donde los propios usuarios/consumidores tomaran conciencia de algún tipo de bienestar humano, logrado a raíz de la utilización de menos recuros naturales o la reutilización de productos.

Para el desarrollo del presente estudio, y siguiendo a Beuren et al (2013), se toma como principal objetivo de la desmaterialización, el de mejorar el bienestar de la sociedad, mediante el desarrollo más eficiente y sostenible, en donde, y coincidiendo con Baines et al., (2007), la desmaterialización sea una oportunidad para que sistemas producto-servicio, rompan el vínculo entre el valor entregado al cliente/usuario y la cantidad de material físico necesario para crear ese valor.

\subsubsection{Digitalización o informacionalización (de átomos a bits)}

El principio que se expone a continuación, pueden ser reconocido por otros nombres como Transmaterialización y Servicing (prestación de servicios), pero se ha considerado que los conceptos de "digitalización" e "informacionalización”, son los que se aproximan más al principio que se propone, a efectos del presente estudio, para ello se ha partido de diferentes aproximaciones teóricas. 
Singh (2002) afirma, que en la era industrial, la atención estaba enfocada en los bienes tangibles, pero que en la era postindustrial, la atención se centra en la producción y el uso de bienes intangibles, relacionados con la información y el conocimiento. Del mismo modo, sostiene que en la era industrial la persona promedio estaba más preocupada por los bienes materiales, pero que en la emergente sociedad de la información, la persona promedio está más interesada en aspectos psicológicos y espirituales de la existencia, afirmando que de esta manera, mediante la digitalización se ha pasado "de átomos a bits".

Shedroff (2009) por su parte propone, que con la informacionalización se pueda replantear un problema y su contexto, en donde por medio de la reducción de recursos se logre convertir "algo en casi nada", a modo de buscar como objetivo principal el tratar de enviar mensajes, recetas, datos, etc. cuando sea y donde sea, para que ese algo en sí mismo -material o inmaterial-, pueda ser replicado en el destino.

En el contexto del diseño de productos y de acuerdo a planteamientos como los propuestos por Vezzoli \& Manzini (2008), referentes a la digitalización de productos o algunos de sus componentes; Garetti, Rosa, \& Terzi (2012), proponen una optimización general para alcanzar una condición más sostenible, la cual sólo puede obtenerse mediante la acumulación y la eficiente gestión de un profundo conocimiento de todo el ciclo de vida del sistema, y la implementación de herramientas avanzadas.

En esta misma dirección, Stevels (2007) afirma que mediante los avances en las tecnologías y la digitalización, se fortalece la entrega de más funciones por unidad de carga ambiental. Lo cual puede ser aprovechado a nivel de producto y servicio, con el fin de sustituir la Tecnología Mecánica (TM) por Tecnologías de la Información (TI) y Tecnología Óptica (TO) o combinar TM, TI y TO de manera inteligente. Esto ya está sucediendo con los actuales Smartphones y Tablets, así como con la transformación de productos en servicios, lo cual, además de haber generado una revolución frente al diseño clásico, disminuye notablemente las cargas ambientales, y va en línea con el aumento de satisfacción emocional del usuario/consumidor.

Desde esta perspectiva, y con base en los anteriores planteamientos, a continuación se nombrarán algunos ejemplos del principio de digitalización -informacionalización-, en donde algunos productos y servicios están desapareciendo y otros han cambiado su estado en bits a partir de átomos. En la música, por ejemplo, lo que anteriormente eran medios fisicos como, discos, casetes, discos compactos, han sido desplazados por la música digital, y en la misma línea se pueden nombrar:

- El video digital, como tecnología de grabacion de imágenes

- El correo electrónico, como servicio de envío y recepción de mensajes y archivos digitales (documentos, imágenes, audios, videos, etc.)

- Los libros digitales (eBooks), como versión electrónica o digital de un libro

- La fotografía digital, como reemplazo a la fotografía química

- Los documentos digitales, que ganan terreno sobre los impresos

- Las herramientas de diseño asistido por ordenador (CAD, CAE, CAM) ${ }^{10}$, que permiten la simulación y pruebas virtuales, de productos modelados digitalmente.

Aunque en estos ejemplos, se ha cambiado el estado de los elementos de atomos a bits, para que haya una interfaz entre el usuario/consumidor y el elemento se necesita de un medio que lo permita, sea este: un ordenador, reproductor de música, teléfono móvil, tablet, televisor, etc.; tamnién es importante resaltar,

\footnotetext{
${ }^{10}$ Por sus siglas en Inglés, Computer Aided Design (CAD), Computer Aided Manufacturing (CAM), Computer Aided Engineering (CAE)
} 
Diseño inmaterial. Hacia la desmaterialización y digitalización de productos y servicios como herramienta de sostenibilidad.

que puede haber oportunidades en donde estos elementos, se conviertan en material físico por acciones como una impresión, un revelado o una grabación.

\subsection{Relación de las necesidades complementarias y las emociones con la sostenibilidad}

Debido al enfoque del presente estudio, se reconocen otro tipo de necesidades complementarias de las personas como usuarios/ consumidores de productos y servicios, para indagar cómo diversas motivaciones y aspiraciones, que no son sólo necesidades básicas -como el beber alguna marca en especial de bebida en lugar de solamente agua-, son aspiraciones o motivaciones que se pueden dar en función de la sostenibilidad. Para analizarlo se recurre a los planteamientos de Maslow (1966), Max-Neef (1992) y Jackson \& Marks (1999) sobre las necesidades, las formas de satisfacerlas y sus escalas o jerarquías.

Wigum (2004), basada en las nueve necesidades humanas fundamentales propuestas por Max-Neef (1992), afirma que estas se pueden dividir en materiales (subsistencia y protección) y no materiales (afecto, entendimiento, participación, ocio, creación, identidad y libertad), y que al menos en parte, pueden estar satisfechas por tanto satisfactores ${ }^{11}$ materiales como no materiales.

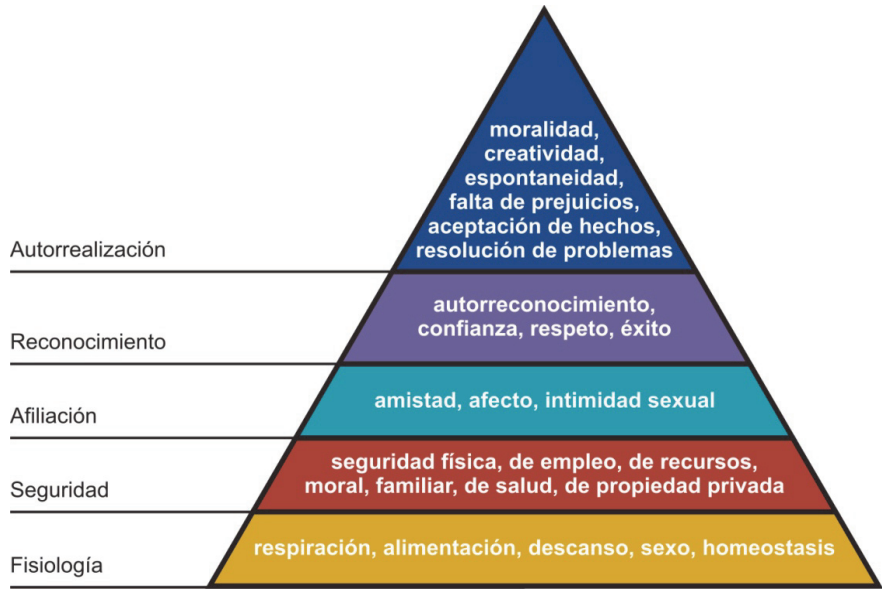

Fig. 3 Pirámide de Maslow - jerarquía de necesidades. Fuente: adaptado de Bartiaux et al (2011)

Por otro lado, se ha tomado como referencia la clasificación de necesidades de Maslow (1943), derivando que, gran parte de los componentes del contexto inmaterial que se relacionan con la sostenibilidad, se encontrarían en la parte superior de la jerarquía de necesidades. Estas necesidades de reconocimiento, pertenencia y autorrealización pueden estar relacionadas con aspectos emocionales, afectivos, espirituales y valores pertenecientes a la dimensión inmaterial en búsqueda de la sostenibilidad.

Para analizar la dimensión emocional, se toma el concepto de "la experiencia del producto" de Desmet \& Hekkert (2007), el cual emplean para referirse a todas las posibles experiencias afectivas involucradas en la interacción producto-humano. Ellos afirman que la interacción producto-humano no sólo se refiere a la interacción instrumental, sino también a la no instrumental, e incluso la interacción no-física. En ese mismo sentido, Nagamachi (1995) afirma, que los consumidores son exigentes en la elección de los productos en términos de su demanda y preferencias. En la actualidad los consumidores son más sofisticados y desean que los productos se ajusten a sus propios sentimientos de diseño, funcionalidad y precio. Un ejemplo de ello ocurre en Japón, donde basados en el “Valor KANSEI” (2007), se pregunta a

\footnotetext{
${ }^{11}$ Los satisfactores, son formas de ser, tener, hacer y estar, de carácter individual y colectivo, conducentes a la actualización de necesidades.
} 
usuarios/consumidores comunes, sobre sus necesidades y recomendaciones para desarrollar productos o servicios que despierten emociones, empatía o resonancia simpática. En este sentido, Vezzoli \& Manzini (2008) afirman, que al tener en cuenta la demanda de satisfacción en nuevos sistemas producto-servicio, se ofrecen diferentes -y más sostenibles- formas de obtener resultados, que podrían convertirse en socialmente apreciados y al mismo tiempo radicalmente favorables para el medio ambiente.

Planteamientos como los anteriores, sobre la relación de algunas necesidades humanas y emociones con la sostenibilidad, apoyan el enfoque que se propone sobre aspectos pertenecientes a un contexto inmaterial, del sistema exterior del MDC, abordados desde una perspectiva sistémica.

\section{Metodología - Planteamiento metodológico}

El presente estudio es realizado de forma descriptiva para analizar, si dinámicas de desmaterialización y digitalización o informacionalización de productos y servicios están relacionadas con la satisfacción de las actuales motivaciones (necesidades, emociones y valores) en los usuarios/consumidores. Con base en un trabajo anterior de los autores, y desde una perspectiva sistémica en la que se establecieron un contexto material y un contexto inmaterial, como derivaciones del sistema exterior del MDC; se pretende establecer si hay una conexión de estas dinámicas y las actuales motivaciones de los usuarios/consumidores con la sostenibilidad.

La investigación es descriptiva correlacional, para lo cual, basándose en investigaciones y teorías sobre actuales dinámicas de desmaterialización y digitalización de productos y servicios, así como en estudios sobre las necesidades del ser humano (Maslow 1966, Max-Neef 1992, Jackson \& Marks 1999), las emociones y la relación usuario-producto (Desmet \& Hekkert 2007, Mugge et al, 2007, Vezzoli \& Manzini 2008), por medio del análisis de un estudio de caso, se logre establecer si estos factores pueden estar relacionados con la sostenibilidad, desde un contexto inmaterial, en el cual se busque llegar a productos cada vez más desmaterializados y digitalizados que afecten menos el medio ambiente.

\section{Estudio de caso}

En la actualidad, algunos productos han desaparecido (VHS, Betamax, casetes de música, máquinas de escribir, etc.), mientras que otros han sido reemplazados por un solo dispositivo (teléfonos, videograbadoras, reproductores de música, calculadoras, GPS, grabadoras, etc.). Aunque actualmente se siguen fabricando muchos de estos productos, se debe resaltar que algo ha cambiado en algunos de ellos; han evolucionado de cómo eran anteriormente en términos de volumen y peso. Los libros se siguen fabricando, a pesar de los ebooks o la enciclopedia británica, después de haber sido reconocida como algo icónico durante más de dos siglos, desaparece como -hecho- medio material y evoluciona hacia un medio virtual e inmaterial, disponible en la red como una nueva vía de acceder al conocimiento.

Con base en las anteriores teorías y planteamientos, como estudio de caso, se establece que el contexto inmaterial puede estar presente en la cotidianidad, sin ser percibido. Para ello, como ejemplo práctico se plantea el siguiente supuesto en el que:

Una persona puede estar retirada de la ciudad, por decir algo, a 50 kilómetros de la zona urbana en un entorno rural, $y$ desde ahi acceder a una serie de servicios que le brindan las funciones y aplicaciones de su Smartphone.

En tiempos pasados esto era muy difícil, por no decir imposible de lograr, debido a que en algunos dispositivos, su volumen, su peso o su conectividad eran un impedimento (teléfono, contestador, 
Diseño inmaterial. Hacia la desmaterialización y digitalización de productos y servicios como herramienta de sostenibilidad.

ordenador, televisor, reproductor de música, radio, etc.), donde en el mejor de los casos, se debían elegir algunos de estos objetos/elementos para llevar consigo. Hoy en día, esta situación ha cambiado, ya que en algunas ocasiones con solamente un dispositivo es posible: hacer llamadas, tomar fotos, grabar videos, escuchar música, jugar, ver películas y programas de Tv, así como conocer las condiciones atmosféricas y la geolocalización del lugar en el que se está; todo esto sin contar, que con el desarrollo de nuevas aplicaciones se amplía el espectro de funciones de este tipo de dispositivos.

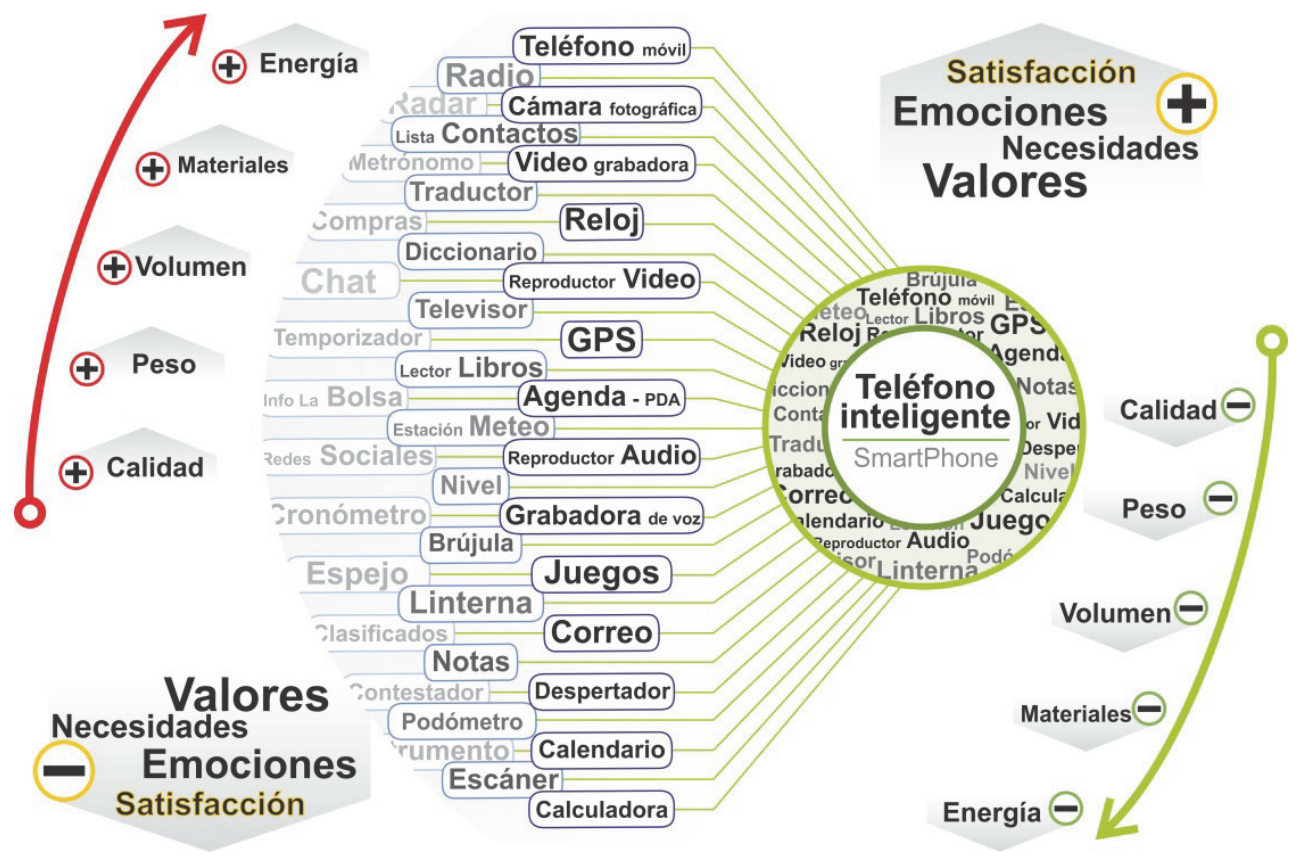

Fig. 4 Desmaterialización y digitalización de productos y servicios. Fuente: elaboración propia

Lo que se pretende con el anterior supuesto, es reafirmar que hay una contexto inmaterial que está presente y tiene una relación directa con la plenitud, las necesidades y las motivaciones reales de la gente, en donde la desmaterialización y la digitalización o informacionalización de productos y servicios contibuyen al desarrollo de todo este fenómeno. Esto se refuerza con el planteameinto de otro caso hipotético en el cual:

La misma persona, que se retira a 50 kilometros del entorno urbano, pero suponiendo que disponga del dinero y los medios de desplazarse con esos equipos fisicos e independientes enrtre sí (teléfono, contestador, televisor, reproductor de música, reproductor de video, radio, calendario, etc.), podría generar aspectos negativos en relación con la plenitud de la experiencia y sus motivaciones, además del impacto negativo que causaría en el medioambiente.

Es importante en este punto, hablar de experiencia, satisfacción y bienestar, pues si se evalúa objetivamente a la persona que se desplaza los 50 kilometros de la ciudad, en estos términos, tendría que cargar con "X kilos de productos", para suplir las mismas necesidades/funciones con algo que pesa alrededor de 140 gramos. En el primer supuesto, no tiene lugar el evaluar la calidad de algunas de las funciones que suple un sólo dispositivo, porque es evidente que la calidad de un Tv plasma, es mucho mejor, al igual que las fotografías que se pueden lograr con una cámara réflex; aquí se trata de lo básico, 
de las funciones básicas y cómo ellas en su conjunto pueden generar una mayor experiencia, satisfacción $\mathrm{y}$ bienestar en el usuario con un menor peso y volumen.

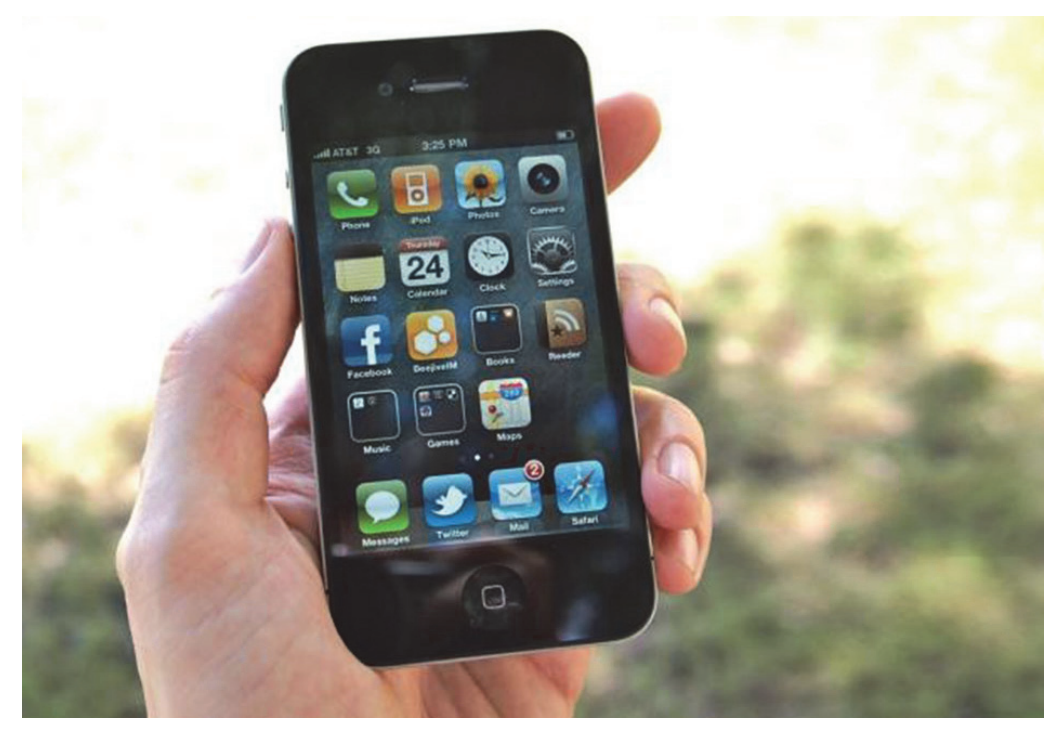

Fig.5 Caso de diseño - teléfono inteligente (iPhone 4). Fuente: (iMore, 2016)

Al respecto, Wahl \& Baxter (2008), citando a Buchanan (1992) destacan el que el poder creativo detrás del pensamiento de diseño se encuentra en "pasar a la modalidad de imposibilidad", y reconocer que lo imposible "realmente sólo puede ser una limitación de la imaginación que puede ser superada por el mejor pensamiento del diseño". Además, también sugiere que el pensamiento de diseño en este contexto es "no pensar dirigido hacia una "solución tecnológica rápida" en hardware, sino hacia nuevas integraciones de signos, cosas, acciones y el ambiente que aborden las necesidades concretas y los valores de los seres humanos en diversas circunstancias". Para ello, es necesario analizar la relación entre los aspectos motivacionales de las personas y lo que caracteriza a la sostenibilidad "hoy en día", para encontrar las verdaderas motivaciones en el uso de productos y servicios y "no" lo que las empresas suponen o sugieren. En este sentido, se podría seguir adelante y cambiar la perspectiva, olvidando el producto o servicio en sí mismo y pensar acerca de las funciones que los usuarios/consumidores necesitan.

\section{Discusión}

La dimensión inmaterial en productos y servicios, es una realidad y está presente en la actualidad. No se puede dar por sentado, a priori, que la gente conoce el concepto "inmaterial", se cree que la gente puede comprender que hay un contexto material y un contexto inmaterial, no solamente porque esté de acuerdo o no, sino porque efectivamente está ocurriendo. Hay un proceso de desmaterialización y de cubrir más necesidades con menos productos, lo cual puede ser evidente en los sistemas producto-servicio. Como ejemplo de ello están, Ascensores Schindler que cambian a vender servicios de transporte vertical en lugar de ascensores; o la empresa Rank Xerox, quienes ofrecen servicios de reproducción a la medida del cliente en vez de vender solamente fotocopiadoras (Stahel, 1998). Este fenomeno se presenta en la actualidad desde empresas que cambian de ofrecer y vender productos a ofrecer servicios, hasta los actuales smartphones y tablets, que integran varios elementos y productos, mediante sus aplicaciones en 
Diseño inmaterial. Hacia la desmaterialización y digitalización de productos y servicios como herramienta de sostenibilidad.

uno solo. Esta nueva forma de interpretar el actual desarrollo de productos y servicios, está basado en anañlizar "lo que la gente quiere y aspira", así como "el por qué lo necesita". Aquí cuando se habla de aspiraciones, necesidades, motivaciones, se refiere a lo que motiva a la gente a adquirir algo, lo cual va en línea con esa dimensión inmaterial.

Volviendo al caso de Xerox, la marca no desarrolla su estrategia desde la óptica de la sostenibilidad, sino desde una perspectiva de negocio mediante la cual pudieran mejorar sus servicios, y que al mismo tiempo esto se viera reflejado en el aumento de sus ingresos; al parecer sin darse cuenta, que ese modelo al mismo tiempo favorece la sostenibilidad.

... ahora, según el actual desarrollo de las cosas/objetos/dispositivos, la relación de los usuarios, con base en sus aspiraciones, necesidades y motivaciones, y reconociendo la existencia de ese contexto inmaterial y su posible relación con la sostenibilidad, es necesario preguntar:

¿La generación de menos objetos pero con más funciones, realmente estaría relacionado con la sostenibilidad?

¿Tienen los procesos actuales de desmaterialización y digitalización o informacionalización de productos y servicios, una relación con una mayor sensación de plenitud de esas funciones?

\section{¿Cuál es la tendencia de la sostenibilidad, en relación con esa valoración de lo inmaterial?}

Desde el punto de vista de tendencias, se cree que hay una tendencia hacia la dismiución productos, pues anteriormente eran necesarios más productos que satisficieran -cubrieran- las necesidades de la gente, mientras que hoy en día, en algunos casos esta satisfacción se puede alcanzar con un solo producto (multifuncionales, smartphones, tablets, etc.). Lo anterior se puede traducir en que, hoy en día , "con menos productos, la experiencia individual aumenta, porque se pueden realizar muchas actividades, mediante las funciones y servicios integrados en un solo elemento". Muchas cosas cambian de formato y dejan de ser tangibles, para convertirse en intangibles.

\section{Conclusiones}

A través del desarrollo de los contenidos tratados, se asume que el aporte del presente estudio, además de reconocer la existencia de un contexto material y un contexto inmaterial desde una perspectiva sistémica, es el de identificar desde la intangibilidad, algunos puntos clave generadores de sostenibilidad. Para este propósito, se ha analizado cómo ésta realidad del contexto inmaterial, que además es tendencia, puede apoyar la sostenibilidad, por el "sólo hecho" de ser características, aspectos, rasgos a los que la gente le da valor; a modo de alcanzar las expectativas y motivaciones más profundas de los usuarios, lo cual puede coadyuvar a que se demande mucho más la sostenibilidad.

Anteriormente los usuarios experimentaban una fragmentación de la relación uso-función con los productos/cosas, esto significa que un producto realizaba "una y sólo una función" por lo que eran necesarios más productos que realizaran funciones específicas; esto si se toma desde la perspectiva de la sostenibilidad significaría la utilización de más materiales y por consiguiente, más volumen y más peso; lo cual a su vez, hacía que la experiencia individual fuera menor porque estaba más fragmentada. En la actualidad se presenta un fenómeno opuesto, en donde, con menos productos se puede alcanzar una mayor experiencia individual; ya que en algunos casos sólo un dispositivo, puede abarcar un mayor número de los dispositivos, productos o elementos que en otros tiempos se utilizaban; lo cual está directamente relacionado con la sostenibilidad. Resultado de lo anterior se concluye lo siguiente: 
"Hay una relación directa de la desmaterialización y digitalización o informacionalización de productos y servicios con las motivaciones (necesidades, emociones y valores) de los usuarios; en donde, por medio de una reducción de materiales - volumen y peso-, un menor consumo de materias primas y energía en producción y uso, además de la optimización funcional -entre otros-, se favorece la sostenibilidad, y al mismo tiempo componen ese contexto inmaterial que involucra aspectos emocionales de satisfacción y bienestar de los usuarios".

Por último, aunque se reconoce una dimensión inmaterial que está relacionada con las necesidades, aspectos emocionales $\mathrm{y} / \mathrm{o}$ valores de los usuarios/consumidores, en donde dinámicas de desmaterialización y digitalización o informacionalización han contribuido a la desaparición de algunos productos, mientras que otros, han evolucionado pasando de ser elementos materiales a ser elemenntos inmateriales o servicios, es probable que en algún momento de la interfaz PRODUCTO/SERVICIOHUMANO, sea necesario tener en cuenta elementos materiales (p. ej. teléfono móvil, tablet, ordenador, televisor...) como medios que permitan la interacción entre el humano y el producto o servicio, así como otro tipo de conexiones (p. ej. energía, internet, datos, fibra...) que consoliden la plenitud de la experiencia y la satisfacción de las demandas, necesidades, emociones y valores de los usuarios/consumidores. Lo anterior refleja que desde un enfoque de diseño inmaterial, así como de dinámicas de desmaterialización y digitalización o informacionalización de productos y servicios, se contribuye a la redución del impacto ambiental causado por el consumo intensivo de elementos materiales.

\section{Referencias}

BAINES, T. S., LIGHTFOOT, H. W., EVANS, S., NEELY, A., GREENOUGH, R., PEPPARD, J., \& ALCOCK, J. R. (2007). "State-of-the-art in product-service systems". Proceedings of the Institution of Mechanical Engineers, Part B: Journal of Engineering Manufacture, 221(10), 1543-1552.

BARTIAUX, F., FROGNEUX, N., \& SERVAIS, O. (2011). "Energy "Needs", Desires, and Wishes: Anthropological Insights and Prospective Views". En F. Sioshansi, \& F. P. Sioshansi (Ed.), Energy, Sustainability and the Environment - Technology, Incentives, Behavior (págs. 63-87). Elsevier Inc. .

BEUREN, F. H., FERREIRA, M. G., \& MIGUEL, P. A. (2013). "Product-service systems: a literature review on integrated products and services". Journal of Cleaner Production, 47, 222-231.

BREZET, H., \& VAN HEMEL, C. (1997). Ecodesign. A promising approach to sustainable production and consumption. France: United Nations Environmental Programme (UNEP).

BUCHANAN, R. (1992). Wicked problems in design thinking. Design issues, 8(2), 5-21.

CHAPMAN, J. (2009). Design for (Emotional) Durability. Design Issues, 25(4), 29-35.

CLEARY, J. (2010). The incorporation of waste prevention activities into life cycle assessments of municipal solid waste management systems: methodological issues. The International Journal of Life Cycle Assessment, 15(6), 579589.

DESMET, P., \& HEKKERT, P. (2007). Framework of product experience. International Journal of Design, 1(1), 5766.

GARETTI, M., ROSA, P., \& TERZI, S. (2012). Life Cycle Simulation for the design of Product-Service Systems. Computers in Industry, 63, 361-369.

GONZÁLEZ, J. R. (2013). El diseño impulsado por la experiencia desde la comunicación multidimensional y la cocreación de valor, en el marco de la interacción empresa-personas. Valencia, España: Repositorio de Biblioteca y 
Diseño inmaterial. Hacia la desmaterialización y digitalización de productos y servicios como herramienta de sostenibilidad.

Documentación Científica, Universidad Politécnica de Valencia. Identificador: http://riunet.upv.es/handle/10251/31524. Obtenido de http://riunet.upv.es/handle/10251/31524

GUILLEN-ROYO, M. (2010). Realising the 'wellbeing dividend': An exploratory study using the Human Scale Development approach. Ecological Economics, 70, 384-393.

HERNANDIS, B. (2003). Desarrollo de una metodología sistémica para el diseño de productos industriales. Valencia, España: Universidad Politécnica de Valencia.

IMORE. (05 de 05 de 2016). www.imore.com. Recuperado el 05 de mayo de 2016, de http://www.imore.com/iphone4

KESTEMONT, B., \& KERKHOVE, M. (2010). Material flow accounting of an Indian village. Biomass and bioenergy, 34(8), 1175-1182.

LI, M., ZHANG, H., LI, Z., \& TONG, L. (2010). Economy-wide material input/output and dematerialization analysis of Jilin Province (China). Environmental monitoring and assessment, 165((1-4)), 263-274.

LÖBACH, B. (1981). Diseño Industrial. Barcelona: Gustavo Gilí, S. A.

MASLOW, A. H. (1943). A Theory of Human Motivation. Psychological Review, 370-396.

MAX-NEEF, M. (1992). Development and human needs. En P. Ekins, \& M. Max-Neef, Real-Life Economics: Understanding Wealth Creation (págs. 197-213). London: Routledge.

MINISTRY OF ECONOMY, TRADE, AND INDUSTRY OF JAPAN. (05 de 2007). "KANSEI" InitiativeSuggestion of the fourth value axis. Recuperado el 22 de 04 de 2014, de http: //www.meti.go.jp/english/information/downloadfiles/PressRelease/080620KANSEI.pdf

MUGGE, R., SCHOORMANS, J. P., \& SCHIFFERSTEIN, H. N. (2007). Product attachment: Design strategies to stimulate the emotional bonding with products. En P. Hekkert, \& H. N. Schifferstein, Product Experience (págs. 42544). Elsevier.

MUNARI, B. (1981). ¿Cómo nacen los objetos? Barcelona: Gustavo Gilí, SA.

NAGAMACHI, M. (1995). Kansei Engineering: A new ergonomic consumer-oriented tech-nology for product development. International Journal of Industrial Ergonomics, 15, 3-11.

ORR, D. W. (2002). The Nature of Design: Ecology, Culture, and Human Intention. Oxford: Oxford University Press.

RIVERA, J. C., GONZÁLEZ, J. R., \& HERNANDIS, B. (2013). Analysis of contexts and conceptual variables for a sustainable approach into systemic model. Relating Systems Thinking \& Design 2013 - Emerging Contexts for Systemic Design. Oslo.

ROBÈRT, K. H., SCHMIDT-BLEEK, B., DE LARDEREL, J. A., BASILE, G., JANSEN, J. L., KUEHR, R., \& WACKERNAGEL, M. (2002). Strategic sustainable development—selection, design and synergies of applied tools. Journal of cleaner production, 10(3), 197-214.

SHEDROFF, N. (2009). Design Is the Problem: The Future of Design Must be Sustainable. Brooklyn, New York: Rosenfeld Media, LLC.

SIDIROPOULOS, E. (2013). Education for sustainability in business education programs: a question of value. Journal of Cleaner Production, xxx, 1-16.

SINGH, J. (2002). From Atoms to Bits: Consequences of the Emerging Digital Divide in India. The International Information \& Library Review, 34(2), 187-200.

STAHEL, W. R. (1998). Transición de productos a servicios: Venta de prestaciones en lugar de venta de bienes. The IPTS Repon, 27, 40. The IPTS Report, 27(40), 35-44. 
STEGALL, N. (2006). Designing for Sustainability: A Philosophy for Ecologically Intentional Design. Design Issues, 22(2), 56-63.

STEVELS, A. (2007). Adventures in EcoDesign of Electronic Products: 1993 - 2007 (Vol. Volume 17 of Design for sustainability program publication). Delft, The Netherlands: Delft University of Technology.

THORPE, A. (2010). Design's Role in Sustainable Consumption. Design Issues, 26(2), 3-16.

UDO, V. E., \& JANSSON, P. M. (2009). Bridging the gaps for global sustainable development: A quantitative analysis. Journal of Environmental Management , 90, 3700-3707.

VEZZOLI, C., \& MANZINI, E. (2008). Design for Environmental Sustainability. London: Springer-Verlag.

WAHL, D. C., \& BAXTER, S. (2008). The Designer's Role in Facilitating Sustainable Solutions. Design Issues, $24(2), 72-83$.

WALKER, S. (2006). Sustainable By Design: Explorations in Theory and Practice. London: Earthscan.

WALKER, S. (2013). Design and Spirituality: Material Culture for a Wisdom Economy. Design Issues, 29(3), 89107.

WIGUM, K. S. (2004). Human and ecological problem solving through radical design thinking - analyses and development of design theory and design framework based on long term human needs and ecological sustainable principles. Trondheim, Norway: Norwegian University of Science and Technology. 